\title{
Improving mental healthcare for ethnic minorities
}

\author{
Kwame McKenzie
}

\begin{abstract}
Multicultural societies offer a significant challenge to mental health services. Different groups have different rates of illness, illness models, ideas of what a suitable pathway of care is and what suitable care looks like. Trying to set up services to meet all these needs can be difficult. There may need to be modifications in clinical practice, service configuration and the way services are commissioned. Ethnic minority communities face complex problems and, consequently, strategies to deal with them can be complex, requiring support from the non-statutory sector, social services and other branches of medicine. Service development often needs research, staff training, race-equality schemes and sufficient funding to make change possible. I offer here a scheme for considering how to think through service development in this area as well as introducing the government strategy, Delivering Race Equality.
\end{abstract}

Mental health services are developed to cater for the dominant economic or cultural group. In practice this means that ethnic minorities do not receive equitable care.

In the UK, disparities in rates of illness, access to care, outcome and satisfaction with services have been reported (Sashidharan, 2003). However, more often than not such disparities are not measured and ethnic minorities suffer in silence.

The problems faced are different for different ethnic groups. This reflects differences in culture, socio-economic status and how they fit within the dominant society (Sashidharan, 2003). However, there are some similarities due to the generally lower socio-economic status of ethnic minority groups, varying degrees of social isolation, acculturation, the impact of migration, and differences in illness models between groups and the services.

Clearly, specific analysis is needed for each ethnic minority group if service development is to be effectively targeted. Here, I will use the situation of mental health services in England as a case study highlighting an approach to service development.

The structure of this article follows the stages that may be appropriate for service re-engineering:

- demography

- needs assessment

- assessment of service gaps

- development strategy.

\section{Demography}

According to the national 2001 census, $91 \%$ of the 49 million people living in England assign themselves to the census population of 'White'. Within this group there are 1.3 million people who state that they are 'White other' and 624000 who say they are 'Irish'. Overall, 9\% of the population are from Black and minority ethnic groups (Table 1) (Office for National Statistics, 2003). Half the people in England who assign themselves to an ethnic minority group were born in England.

\begin{tabular}{lr|}
$\begin{array}{l}\text { Table } 1 \text { The nine largest Black and minority ethnic } \\
\text { groups in the UK }\end{array}$ \\
\hline South-Asian Indian & 1029000 \\
South-Asian Pakistani & 707000 \\
South-Asian Bangladeshi & 275000 \\
South-Asian Other or White Asian mixed & 422000 \\
Black Caribbean & 561000 \\
White and Black Caribbean mixed & 231000 \\
Black African & 476000 \\
Chinese & 221000 \\
Other ethnic group & 215000 \\
\hline 1. Figures rounded to nearest 1000. &
\end{tabular}

Kwame McKenzie is Professor of Psychiatry at the University of Toronto, Medical Director for Diversity and a senior scientist in social equity and health research at the Centre for Addictions and Mental Health, Toronto (CAMH, 455 Spadina Avenue, Suite 300, Toronto, Ontario M5S 2G8, Canada. Email: Kwame_McKenzie@camh.net) and a Professor of Mental Health and Society at the University of Central Lancashire. He was part of the team that drafted Delivering Race Equality. 
As a whole, White minority ethnic groups and Black and minority ethnic groups experience high levels of social and material deprivation. The social exclusion of ethnic minority groups is complex and varies according to economic, social, cultural and religious backgrounds (e.g. Commission for Racial Equality, 2006).

The ethnic groupings of the 2001 census have some administrative use but are not culturally coherent. The Black African group, for instance, includes people from many different countries, with diverse languages and cultural beliefs, who are in England for different reasons. It includes economic migrants, refugees, asylum seekers and people of South Asian origin who left Uganda. Because of this, using census population groups to inform service development can be problematic.

\section{Needs assessment}

If it were possible to start from scratch and to set up appropriate services for a multicultural population in an area, a needs assessment would be the first task. Cross-cultural needs assessments can be difficult. A fundamental problem is the need to sample different ethnic minority groups in a way and in sufficient numbers that will produce meaningful answers. Another problem is that even if it is possible to find these samples, there are no tools robust enough to capture the different needs of cultural groups (Winters \& Patel, 2003).

Partly because of this, the literature on crosscultural needs has been dominated by surveys of rates of illness. The most comprehensive assessment is the Ethnic Minority Psychiatric Illness Rates in the Community (EMPIRIC) report (Sproston \& Nazroo, 2002). This study investigated rates of illness in the UK's six largest ethnic groups. It found modest differences of rates of common mental disorders, with Irish men and Pakistani women reporting higher rates, and Bangladeshi women lower rates than same gender White respondents.

Contrary to studies based on service contact (McKenzie et al, 2008), which report a markedly raised incidence of psychotic illness in people in England of African and Caribbean origins and an increased incidence in those of South Asian origin, EMPIRIC did not find a significantly higher prevalence of psychotic illness and reported only a twofold increase in prevalence of psychotic symptoms for the Black Caribbean group compared with the White British population, which was not statistically significant (Sproston \& Nazroo, 2002).

However, comparative illness rates do not assess needs. Although epidemiological surveys may conclude that the incidence or prevalence of an illness is greater in one ethnic group than another after controlling for socio-economic and age differences between groups, it is the actual burden of illness that is important for service development. Local services do not have the luxury of being able to 'control' population structure or socio-economic class. They have to offer services to their population and if that population has multiple problems then the burden of illness and needs will be high.

Moreover, the same illness may be more of a burden on one group than another. For instance, the capacity to support someone with depression depends on individual, community and family resources as well as the other difficulties the community has to deal with: for example, the needs of a refugee or asylum seeker with depression may be magnified by other factors such as the asylum process, social exclusion, isolation and language problems.

Qualitative work (Grewal \& Lloyd, 2002) has demonstrated that those who do not speak English have experiences that are quite different and their needs may be missed by standard surveys. Family tensions are significant but rarely documented, and recent migrants have acute problems with acculturation and negotiating the health system that are not measured. Racism has an important impact on mental health but dealing with it is not considered a health need $\left(\mathrm{O}^{\prime}\right.$ Connor \& Nazroo, 2002). How all of these factors interact with socioeconomic problems and difficulties due to education and housing is rarely considered.

In the field of substance misuse, local communities have developed a technique called 'community engagement' to tackle some of these issues. Local community groups are trained in research methods and report-writing. They are asked to develop a project that will allow sensitive community-based needs assessments. The technique is used to reach communities that usual surveys miss and to give a more accurate reflection of a community's needs. This technique has recently started to be employed in mental health (Department of Health, 2005).

\section{Service gaps}

In my experience, services do not undertake comprehensive needs assessments. This is in part because health systems are pragmatic and not built directly to meet the needs of their communities. The question most people ask when they want to produce equitable services is whether there are specific service gaps - that is, whether particular groups are disadvantaged given the existing configuration rather than whether the existing configuration is cross-culturally valid. 
Despite the higher levels of ill health and disability among ethnic minority groups (Sashidharan, 2003), these groups appear to have poorer access to health services. Headline figures showing increased rates of hospitalisation of people of Caribbean and African origin with psychosis (Healthcare Commission, 2005) and higher rates of detention under the Mental Health Act belie the fact that people from ethnic minority groups who say that they want help have difficulty getting treatment for common mental disorders such as depression and anxiety and in getting any psychological treatment (Sproston \& Nazroo, 2002). Moreover, the lack of adequate interpreting services means that those who do not speak English may have access but do not receive the same treatment.

There is surprisingly little research on the mental healthcare experience of ethnic minority groups in England and on the outcome of treatment. However, from the research that exists, including clinical and epidemiological studies, anecdotal accounts and the testimonies of service users and carers, interested parties have concluded that ethnic minorities have a worse experience of mental healthcare than others (Sashidharan, 2003).

The blueprint for service design in England is the National Service Framework (NSF) for Mental Health (Department of Health, 1999). Although cross-cultural suitability of the NSF has not been assessed, given that it is the model, it can be used as a template for considering service gaps.

The NSF sets out seven national standards for mental health in five key areas: mental health promotion, primary care, access to care, effective services, carers, and suicide. A simple analysis of service gaps in these areas is enlightening.

\section{Standard 1: mental health promotion}

The NSF for Mental Health states that health and social services should promote mental health for all, working with individuals and communities, and that these agencies should combat discrimination against individuals and groups with mental health problems, and promote their social inclusion.

The differences in rates of illness between ethnic minority groups and their different social stresses could indicate different balances of risk factors. For instance, racism is less commonly experienced by White minority ethnic groups than by Black or South Asian groups (Karlsen et al, 2005). Coupled with cultural differences regarding language barriers and access to media, effective mental health promotion would need a comprehensive strategy just for ethnic minorities. As there is no coordinated strategy in England, this could be considered a service gap.

\section{Standards 2 and 3: primary care and access to services}

Standard two of the NSF states that any service user who contacts their primary healthcare team with a common mental health problem should have their mental health needs identified and assessed, and be offered effective treatments, including referral to specialist services for further assessment, treatment and care, if they require it.

The research that is available shows that people from ethnic minorities with common mental disorders are as likely, if not more likely, to have recently seen their general practitioner (GP). However, they are less likely to have been treated for their psychological problem. Many GPs fail to recognise psychological symptoms in ethnic minorities but this is not the whole story (Sproston \& Nazroo, 2002). Some ethnic minority groups are less likely to present their psychological problems to GPs because they do not consider them to be the most appropriate person to treat them (Sproston \& Nazroo, 2002).

Thus, standard 2 is not being met for Black and minority ethnic (the so-called BME) groups, indicating a need for cross-cultural psychological service development in primary care.

Standard three requires that any individual with a common mental health problem should be able to make contact around the clock with the local services necessary to meet their needs. The addition of crisis teams and assertive outreach teams has improved access to services (Killaspy et al, 2008). However, there is next to no research demonstrating whether they have improved access to care for Black and minority ethnic groups. There is no research into whether these services are better able than previous services to make decisions on assessment and possible management options for these groups. As mentioned above, data from the Count Me In census indicate that some ethnic minority groups are more likely to be admitted to hospital than others (Healthcare Commission, 2005). People of African or Caribbean origins are also more likely to be detained against their will. The use of more coercive pathways may indicate that services are not meeting the needs of some groups, that they are not engaging and/ or that they have different pathways of care, with more crisis admissions.

\section{Standards 4 and 5: effective services for people with severe mental illness}

These standards state that anyone with a severe mental illness should receive the range of mental health services they need: crises should be anticipated 
and, if possible, prevented; prompt and effective help should be available if a crisis does occur; and they should have timely access to an appropriate and safe mental health establishment or hospital bed, including a secure bed, as close to their home as possible.

Cross-cultural and cross-ethnic assessment of the effectiveness of services has rarely been undertaken. There is a wealth of data reporting differences in service use and outcome between different ethnic groups (Sashidharan, 2003). However, there is considerable debate as to whether this reflects different rates of illness, different needs, different efficacy of treatment, socio-economic difficulties or service gaps (McKenzie \& Bhui, 2007). That there are differences in the experience of services is not doubted, hence from a human rights and legal perspective there is a need for change. However, developing appropriate and responsive services will require more fine-grained analyses, including a wider understanding of all the elements of the care pathway.

\section{Standard 6: caring about carers}

Health and social services should assess the needs of carers who provide regular and substantial care for those with severe mental illness, and provide appropriate care to meet their needs.

However, given the power imbalance and language problems, it is unlikely that carers from Black and minority ethnic groups receive equal access. Culturally appropriate advocacy services to support and campaign on behalf of service users from ethnic minority communities, and their carers, could facilitate equal access for carers. Such services are often not available and so could be argued to constitute a service gap.

\section{Standard 7: preventing suicide}

England and Wales has a national suicide prevention strategy (Department of Health, 2002). This strategy has been considered important in decreasing the rates of suicide in the general population. Despite research indicating that there are differences in the rates, causes and methods of suicide in different ethnic minority groups (McKenzie et al, 2003a), there is no specific mechanism to make sure that the national strategy meets their needs or is equally effective. This is a clear service gap.

\section{A strategy for service development}

Aneeds assessment may indicate what the morbidity is within the community for different ethnic groups.
The gap analysis may help service developers to compare needs with the existing configuration, as a prelude to the development of a more appropriate service structure or balance. In a perfect world, a root and branch reform of services would then be undertaken, dealing with generic problems as well as problems specific to particular groups. However, this is rarely possible and the development of services is often piecemeal, either on a problem-by-problem or a group-by-group basis. If service reform to meet the needs of ethnic minorities is to be tackled, there must be an overall structure and plan, and a coherent, ethical decision-making process that recognises the need for decisions about resource allocation.

The problem with piecemeal change is that strategies for service development need to work at a number of levels, for example:

- development of clinicians' skills, attitudes and knowledge

- better support for clinicians, e.g. interpreting services and advocacy

- culturally acceptable models of service delivery

- improvements in access to services - pathways into and out of care

- greater influence of the community in the commissioning of secondary care

- support and development of the non-statutory/ voluntary sector

- culturally valid health promotion strategies in public health

- a legislative framework that supports service development

- wider social policy aimed at the determinants of health

- audit to monitor change and outcomes.

The problems are complex, and often the strategies will be complex as well, involving a number of partners. In many cases, service development will need research to expand the knowledge base, training to equip staff with the skills that are required, race-equality schemes to ensure that unlawful discrimination neither hinders organisational development nor affects the patient experience, and sufficient funding to makes change possible.

This is clearly not going to be a quick fix - it requires a long-term strategy. However, in England such a strategy has been developed. A broad consensus on the need for better services for Black and minority ethnic groups led to the development of the Delivering Race Equality in Mental Healthcare (DRE) initiative (Department of Health, 2005). ${ }^{\dagger}$ Delivering

\footnotetext{
${ }^{+}$Delivering race equality in mental health services will be the topic of an Editorial by Annie Lau in the next issue of APT. Ed.
} 
Race Equality builds on a previous report, Inside Outside, which the public and patients helped to formulate (Sashidharan, 2003).

The strategy is targeted at institutions and attempts to develop appropriate and responsive services by working at multiple levels to improve race-equality systems, training in cultural capability (Bhui et al, 2007), and information and research (Department of Health, 2005). It also attempts to engage communities so that they are involved in decision-making and can help in the evolution of new service configurations and pathways into care to evolve (Department of Health, 2005).

However, moving from policy and consensus to implementing sustainable change in mainstream mental health services can be difficult. Research has demonstrated that using the fact that institutions have duties under the Race Relations Amendment Act 2000 is an important driver for producing equal public services for Black and minority ethnic groups (Audit Commission, 2003). The Commission also states that this should be coupled with an understanding of institutional racism (McKenzie \& Bhui, 2007). Because of this, DRE was explicitly linked to the Race Relations Amendment Act 2000.

Delivering Race Equality has three building blocks: appropriate and responsive services, community engagement and better information (Box 1).

The aim of the appropriate and responsive services building block is to make sure that direct clinical care of all Black and minority ethnic groups is improved. The community engagement building block refers to the actions needed (inside and outside mental health services) to give Black and minority ethnic communities genuine opportunities to influence mental health policy and provision, and to promote mental health and recovery. The better information building block sets out action that is needed to make sure that there is better information on service use and needs, and knowledge of recovery-enhancing environments and approaches.

This all sounds very general. In fact, in DRE there are over 70 different specific actions - things that could be done to improve services for ethnic minority groups. The actions are different for different ethnic minority groups and for different problems. They are open to local interpretation.

\section{DRE in practice}

To bring this policy to life, I will focus on a specific problem cited in DRE - people of Caribbean origin with psychosis who consider or attempt suicide.

Research in the 1990s had shown that people of Caribbean origin with a diagnosis of psychosis living in the UK had a five times lower risk of selfharm and suicide compared with their British White

\section{Box 1 The building blocks of the DRE action plan}

Appropriate and responsive services

The action envisaged in this building block aims to:

- develop organisations so that they offer high-quality, non-discriminatory and recovery-oriented healthcare

- develop a workforce that can deliver equitable care to Black and minority ethnic populations

- improve clinical services for Black and minority ethnic populations

- improve services for specific populations, including older people, asylum seekers, refugees, children and adolescents

Community engagement

Actions needed to:

- help build healthier communities

- engage communities, build capacity, deliver services and facilitate change in local mental health service economies

Better information

Actions needed to:

- improve the monitoring of ethnicity and mental health service use

- improve the analysis and dissemination of information

- improve the knowledge available on effective services

- evaluate the impact of the action plan

peers (McKenzie et al, 1995). By the new millennium there were reports that young African-Caribbeans had the same risk as British Whites (McKenzie et al, 2003b). Clinicians in charge of the care of patients who have died by suicide are more likely to report for African-Caribbeans that they believe the suicide could have been prevented than for patients from other ethnic groups (Hunt et al, 2003). Moreover, people of Caribbean origin who attempt suicide are the ethnic group least likely to seek professional or lay help before the attempt (Crawford et al, 2005). How can the building blocks of DRE be used to address this issue?

\section{Appropriate and responsive services}

Under this first building block, a number of actions could be considered. The first would be to scrutinise the clinical encounter, the care received and the engagement of clients (patients). Clients' views of services and the difficulties they have with them 
would need to be considered, and specific service development initiatives to make clients more comfortable with them may be indicated. The data and literature on this issue (e.g. McKenzie et al, 1995, 2003b; Hunt et al 2003; Bhui \& McKenzie, 2008) could be developed into training modules, which would be used to make services aware that there is a problem and that they should be vigilant.

\section{Community engagement}

This second building block may be particularly important. Given that there seems to be a cultural shift towards greater risk of self-harm and suicide among African-Caribbeans, there are likely to be specific stresses and problems within the community that need attention. This will need research. Engaging communities could help not only to identify unmet needs and different models of service provision that may be applicable to the problem, but also to unearth any non-statutory sector expertise that may be better placed to offer support. Other providers such as the Samaritans and whether their services offer equitable care would be discussed. The building of knowledge and links in the community is a way of developing capacity through education and of highlighting the problem and creating networks of people interested in preventing suicide. If there is a need for a public awareness campaign then such a network could help this to be properly targeted with appropriate messages.

\section{Better information}

This third building block is vital. Information on suicide for those in contact with services is available, but ethnicity is not recorded on death certificates in the UK and so suicide rates in the wider population are not available by ethnic group. Hence, we do not know whether this problem simply reflects changes in the wider society or whether it is a specific servicerelated problem. Better information, changes in data recording and a more robust suicide-recording strategy would help in this regard.

That some people are in contact with mental health services but still take their own lives is understandable, though disquieting. The fact that services believe that it is more preventable in people of Caribbean origin argues for better information on why it is not prevented. It requires a programme of research. Is the problem education, attitudes or skills? Are the risk assessment tools used culturally valid or are there risk indicators that clinicians do not know about? What are the risk factors for suicide in those of Caribbean origin?
All the information from such a research effort would need to be fed into publications and training developed to improve services.

\section{Conclusions}

The reader may be uncomfortable with the fact that this is not a 'how to' article. It does not always give direct answers but tries to offer a generic approach. It offers a schema to help in the identification of what knowledge is needed or, more usually, an understanding of what clinicians do not know.

The reason this is not a 'recipe' is that ethnic groups within any country are diverse and changing. There is no 'one size fits all' and cook-book solutions go out of date quickly.

The aim here has been to encourage the reader to understand the complexity of the situation. Clinicians can keep themselves informed and educated - certainly they should all be conversant with clinical issues as well as the socio-cultural history of the largest ethnic minority populations. But clinicians cannot completely make up for the health infrastructure that they work in. They can do their best but for that to be enough they have to understand what else needs to be done and try to help it happen.

\section{Declaration of interest}

None.

\section{References}

Audit Commission (2003) The Journey to Race Equality. Audit Commission.

Bhui, K. \& McKenzie, K. (2008) Rates and risk factors by ethnic groups for suicides within a year of contact with mental health services in England and Wales. Psychiatric Services, 59, 414-420.

Bhui, K., Warfa, N., Edonya, P., et al. (2007) Cultural competence in mental health care: a review of model evaluations. BMC Health Services Research, 7, 15.

Crawford, M. J., Nur, U., McKenzie, K., et al (2005) Suicidal ideation and suicide attempts among ethnic minority groups in England: results of a national household survey. Psychological Medicine, 35, 1369-1377.

Commission for Racial Equality (2006) Employment and Ethnicity (Factfile 1). CRE (http://www.equalityhumanrights.com/ Documents /CRE / PDF / factfile01_employment_and_ ethnicity.pdf).

Department of Health (1999) National Service Framework for Mental Health: Modern Standards and Service Models. TSO (The Stationery Office) (http://www.dh.gov.uk/en/Publications andstatistics/Publications/PublicationsPolicyAndGuidance/ DH_4009598).

Department of Health (2002) National Suicide Prevention Strategy for England. UK Department of Health.

Department of Health (2005) Delivering Race Equality in Mental Health Care. TSO (The Stationery Office).

Grewal, I. \& Lloyd, K. (2002) Use of services. In Ethnic Differences in the Context and Experience of Psychiatric Illnesses: A Qualitative 
Study (eds W. O'Connor \& J. Nazroo), pp. 51-60. TSO (The Stationery Office)

Healthcare Commission (2005) Count Me In. Commission for Healthcare Audit and Inspection (http://www.healthcare commission.org.uk/_db/_documents/04021830.pdf).

Hunt, I., Robinson, J., Bickley, H., et al (2003) Suicides in ethnic minorities within 12 months of contact with mental health services. British Journal of Psychiatry, 183, 155-160.

Karlsen, J., Nazroo, K., McKenzie, K., et al (2005) Racism, psychosis and common mental disorder among ethnic minority groups in England. Psychological Medicine, 35, 1795-803.

Killaspy, H., Johnson, S., King, M., et al (2008) Developing mental health services in response to research evidence. Epidemiologia e Psichiatria Sociale, 17, 47-56.

McKenzie, K. \& Bhui, K. (2007) Institutional racism in mental health care. BMJ, 334, 649-650.

McKenzie, K., van Os, J., Fahy, T., et al (1995) Evidence for good prognosis illness in African-Caribbeans now living in the UK. BMJ, 311, 1325-1328.

McKenzie, K., Serfaty, M. \& Crawford, M. (2003a) Suicide in minority ethnic groups. British Journal of Psychiatry, 183, 100-101.

McKenzie, K., van Os, J., Samele, C., et al (2003b) Suicide and attempted suicide among people of Caribbean origin with psychosis living in the UK. British Journal of Psychiatry, 183, $40-44$

McKenzie, K., Fearon, P. \& Hutchinson, G. (2008) Migration, ethnicity and psychosis. In Society and Psychosis (eds C. Morgan, K. McKenzie \& P. Fearon), pp. 143-160. Cambridge University Press.

O'Connor, W. \& Nazroo, J. (2002) Ethnic Differences in the Context and Experience of Mental Illness: A Qualitative Study. TSO (The Stationery Office).

Office for National Statistics (2003) UK population: by ethnic group: Census update (Spreadsheet). ONS (http://www.statistics. gov.uk/statbase/ssdataset.asp? $v$ lnk $=6588 \&$ More $=\mathrm{Y}$ ).

Sashidharan, S. P. (2003) Inside Outside: Improving Mental Health Services for Black and Minority Ethnic Communities in England. UK Department of Health.

Sproston, K. \& Nazroo, J. (2002) Ethnic Minority Psychiatric Illness Rates in the Community (EMPIRIC) - Quantitative Report. UK Department of Health

Winters, M. \& Patel, K. (2003) Community Engagement Report 1 The Process. University of Central Lancashire.

\section{MCQs}

1 The largest ethnic minority group in the UK are people of:

a Irish origin

b Chinese origin

c Caribbean origin

d African origin

e Indian origin.

2 Compared with the British White population, the EMPIRIC study found higher rates of common mental disorders in:

a Indian men

b Indian women

c young African-Caribbeans

d Pakistani women

e Irish women.
3 Significantly increased rates of psychotic illness have been reported in:

a a UK community prevalence study comparing Black Caribbean men with a UK White ethnic group

b a UK community prevalence study comparing Black Caribbean women with a UK White ethnic group

c UK incidence studies comparing East Asians with people of a White ethnic group

d UK incidence studies comparing people of African origin with people of UK White ethnicity

e UK community prevalence studies comparing people of African origin with people of White ethnicity.

4 The following is true:

a there are many cross-culturally validated tools that can be used to assess mental health needs

$\mathrm{b}$ incidence and prevalence rates are sufficient to assess mental health needs

c in the UK the health needs of those who speak English are similar to those who do not

$d$ the health needs of different ethnic groups are not the same

e the National Service Framework for Mental Health is a cross-culturally valid model for mental health services.

5 Delivering Race Equality is:

a a local NHS policy for improving services for ethnic minorities with mental health problems

b the same as the Race Relations Amendment Act 2000 and has nothing to do with doctors

c a strategy for improving services that tells clinicians exactly what they need to do for each ethnic group

d a way of blaming psychiatrists for problems that are due to poverty

e a policy which has resulted from wider consultation.

\section{MCQ answers}

$\begin{array}{lllllll}1 & & \text { 2 } & 3 & & 4 & 5 \\ \text { a F } & \text { a F } & \text { a F } & \text { a F } & \text { a F } \\ \text { b F } & \text { b F } & \text { b F } & \text { b F } & \text { b F } \\ \text { c F } & \text { c F } & \text { c F } & \text { c F } & \text { c F } \\ \text { d F } & \text { d T } & \text { d T } & \text { d T } & \text { d F } \\ \text { e T } & \text { e F } & \text { e F } & \text { e F } & \text { e T }\end{array}$

